\title{
Longevity enhances selection of environmental sex determination
}

\author{
J. J. Bull and \\ Michael G. Bulmer
}

\author{
Department of Zoology, University of Texas, Austin, \\ TX 78712, U.S.A. \\ Dept. Statistics, University of Oxford, 1 South Parks \\ Road, Oxford OX1 3TG.
}

Environmental sex determination (ESD) is a mechanism in which an individual develops as male or female largely in response to some environmental effect experienced early in life. Its forms range from sex determination by egg incubation temperature in reptiles to sex determination of photoperiod in amphipods. Previous theoretical work as suggested that ESD is favored by natural selection if the fitness consequences of the early environmental experience differ for males and females, so that an individual benefits by being male under some conditions and female under others. A drawback of ESD is that it enables climatic changes to influence the population sex ratio, and such fluctuations select against ESD. This study employed numerical analyses to investigate the balance between these two opposing forces. The negative impact of climatic fluctuations appears to depend greatly on species longevity: substantial between-year fluctuations are of little consequence in selecting against ESD in long-lived species because annual sex ratio fluctuations tend to cancel and thus alter the total population sex ratio only slightly. Thus, if a species is sufficiently long-lived, extreme ESD can be maintained despite only a weak advantage. This result offers one explanation for the failure to demonstrate an advantage for the extreme forms of ESD observed in reptiles.

\section{INTRODUCTION}

The sex determining mechanisms of dioecious species can be divided into two major classes: genotypic sex determination (GSD), in which an individual's gender depends largely on its genotype, and environmental sex determination (ESD), in which gender is influenced appreciably by the larval or embryonic environment (reviewed in Bull 1983; Adams, Greenwood, and Naylor, 1987). The extent to which the sex ratio is affected by the environment under ESD varies from a virtually all-male/all-female effect of temperature in some reptiles to a mild shift in sex ratio per temperature in some fish.

Although ESD and GSD both maintain males and females in a population, they are not generally equivalent under natural selection, and some models have been proposed to suggest advantages of one mechanism over the other (see below). Our current understanding, which is qualitative, is that ESD is favored by some factors whereas GSD is favored by others, and the magnitude of ESD that evolves balances the positive and negative forces. This paper offers a numerical study to determine combinations of parameters that can favor extreme forms of environmental effects on sex determination. Of particular interest in this respect are those reptiles with temperature-dependent sex determination, as there is presently a striking failure to understand the significance of ESD in this group (Bull 1983; Bulmer 1987; Head et al. 1987; Bull and Charnov 1989).

\section{WHY ESD?}

Charnov and Bull (1977) proposed that ESD might be superior to GSD under particular conditions, based on a sex ratio argument of Trivers and Willard (1973). Specifically, ESD is favored if three conditions occur in a life history: (i) the species inhabits a patchy environment, such that offspring raised in some patches develop as relatively better males than females, whereas other patches have the opposite effect; (ii) parents and offspring cannot choose the patch in which sons versus daughters are raised; (iii) individuals raised in one patch are free to mate with individuals raised in other patches. These conditions favor ESD by enabling the individual to develop as the sex that benefits the most in each patch type. GSD would cause 
some females to develop in patches that benefitted males and vice versa. A basic prediction of this model therefore is that species with ESD will have life histories consistent with these three requirements. Many of the known cases of ESD in fact offer at least superficial support of this prediction (Charnov, 1982; Bull, 1983; Conover, 1984; Naylor et al., 1988).

The Charnov-Bull model offers no insight to possible disadvantages of ESD-the model in fact leads us to expect that ESD should be common. A nearly ubiquitous disadvantage of ESD, however, stems from the dependence of population sex ratio on the environment: virtually any environmental factor is likely to vary from year to year, and fluctuation of the sex-determining factor will in turn cause the sex ratio to vary between years. These sex ratio fluctuations in turn select genic control of sex determination (Bull, 1981; Bulmer and Bull, 1982). Only a compensating advantage of ESD, such as that of the CharnovBull model, is therefore likely to maintain any environmental effect on sex determination.

\section{THE PROBLEM POSED BY ESD IN REPTILES}

The most extreme forms of ESD known occur in reptiles whereby the incubation temperature of the embryo determines the sex of the hatchling. For example, map turtles incubated at constant temperatures of $23-28^{\circ} \mathrm{C}$ hatch only as males, those incubated from $30-35^{\circ} \mathrm{C}$ hatch only as females, and the sex ratio changes abruptly from all male to all female over just $2^{\circ} \mathrm{C}$ change in temperature.

The reptiles with ESD do not obviously fit the Charnov-Bull model, yet there is likewise no evidence that they are inconsistent with the model (Bull, 1983; Bulmer, 1987; Head et al., 1987; Bull and Charnov, 1989). There can be little doubt that between-year climatic variation causes the annual sex ratio of hatchlings to fluctuate somewhat, so the major issue is whether embryonic temperature influences fitness differently for males than for females. Reptiles are so long lived that studies of absolute fitness are rarely feasible, but it would be useful to understand the magnitude of temperature effects on fitness that are sufficient to select these extreme levels of ESD: could the observed level of ESD be maintained with a temperature effect on fitness so slight that it would be undetectable?

\section{THE MODEL}

The model studied here follows the outline of some earlier models of ESD (Bull, 1981; Bulmer and
Bull, 1982). A model for the evolution of environmental sex determination requires two variables, one representing the environmental determinant of sex (designated as $T$, for temperature), and one for the characteristics of the species that influences its response to $T$ in becoming male or female (designated as $Z$ ). Each individual is conceived with a value of $Z$, and early in life it is exposed to a particular value of $T$ due to the combined effects of its mother's choice of nest site and to climatic events. The rule of sex determination for each individual is

$$
\begin{aligned}
& T>Z \text { is male } \\
& T \leq Z \text { is female. }
\end{aligned}
$$

Thus, males develop at high values of $T$, females at low ones. Although the values of $T$ and $Z$ are fixed for each individual, different individuals generally have different values.

The population sex ratio and even the form of ESD depends on the joint distribution of $T$ and $Z$ within each year. (We will assume independence of the two variables, with distributions $\alpha(Z)$ and $\phi(T)$, which may vary with time.) If all individuals share the same value of $Z$, the sex ratio will be 100 per cent male at all $T$-values above this threshold and 100 per cent female at $T$-values below the threshold; ESD is thus extreme. Alternatively, if the variance in $Z$ is large relative to the variance in $T$, some individuals will develop as male and others as female at all temperatures; ESD is then weak or absent. In studying the evolution of ESD, therefore, our interest lies in understanding how the variance of $Z$ evolves relative to the variance in $T$.

Appendix I provides details of the model; parameter values were chosen based on considerations of tractability and empirical validity for reptiles. The following list of assumptions provides basic details of the model: (i) $Z$ is assumed to be inherited as a polygenic character, but $T$ is not heritable; (ii) survival from conception to age 1 is proportional to $W_{m}(T)$ for a male and $W_{f}(T)$ for a female raised at $T$; (iii) maturity occurs at age 1, with constant adult survival thereafter; (iv) environmental fluctuations occur as between-year changes in the mean of $T(\bar{T})$.

\section{RESULTS}

A variety of parameter values were studied, but it is expedient to restrict this presentation to three cases, designated as (i) the Basic model, (ii) the Short-Lifespan model, and (iii) the Autocorrelated $-\bar{T}$ model, with results illustrated in fig. 1 . All 

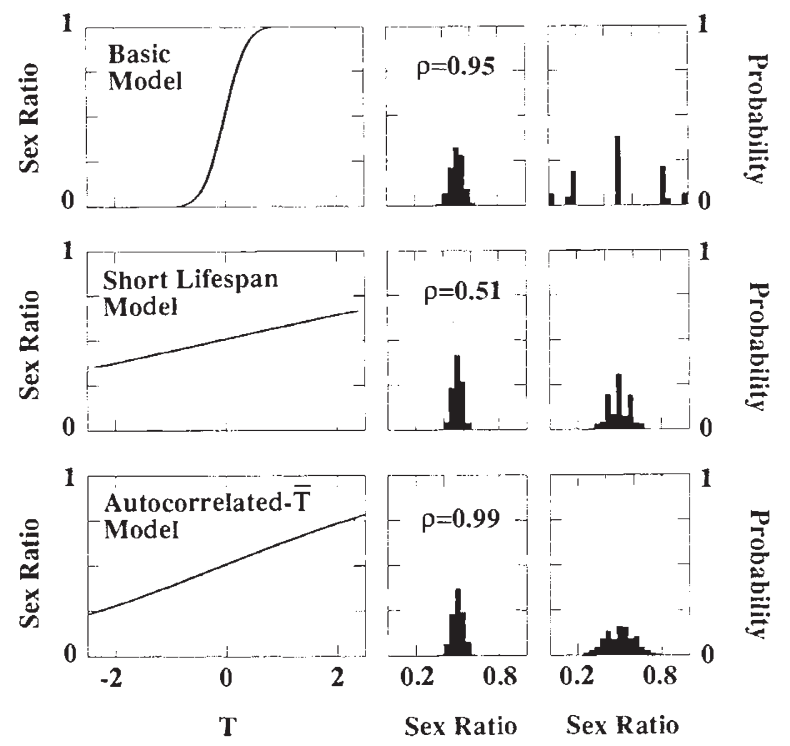

Figure 1 Evolution of ESD. Representative results for the Basic model (top row), Short-Lifespan model (middle row), and the Autocorrelated- $\bar{T}$ model (bottom row) are compared (see text and Appendix 1 for the parameterizations of these models). The left figure in each row represents the form of ESD versus $T$ at equilibrium, which is most extreme in the Basic model and least extreme in the Short-Lifespan model. The scale may be treated as ${ }^{\circ} \mathrm{C}$ centered on some intermediate temperature. The second figure in each row presents a summary histogram of the cumulative population sex ratio observed at equilibrium. The autocorrelation of the sex ratio from one year to the next is given in the figure as $\rho$, and is almost perfect for the model of Autocorrelated- $\bar{T}$. Rightmost in each row is a histogram of the frequency of annual primary sex ratios at equilibrium, which is extremely variable in the Basic model. The histograms in the middle and right columns were calculated for trials of 5000 years at equilibrium conditions; sex ratios were lumped into intervals of 0.04 . The distributions of primary sex, ratio have several discrete peaks because the environmental state (which in turn influenced the primary sex ratio) was chosen as a discrete variable. Fitness functions used in these trials are shown fig. 2 .

three models employed identical male survival functions, $W_{m}(T)$, identical female survival functions, $W_{f}(T)$, and the same distribution of $T$ within years. The male survival function was the same shape as the female survival function, but its optimum exceeded that of the female function by 0.4 units (fig. 2). The three models therefore differed only in adult survival and in the nature of environmental fluctuations. (i) The Basic model assumed an adult survival of 0.95 (average reproductive life-span of 20 years) and that $\bar{T}$ was chosen at random each year. (ii) The Short-Lifespan model assumed an adult survival of 0.6 (an average reproductive lifespan of $2 \frac{1}{2}$ years) with $\bar{T}$

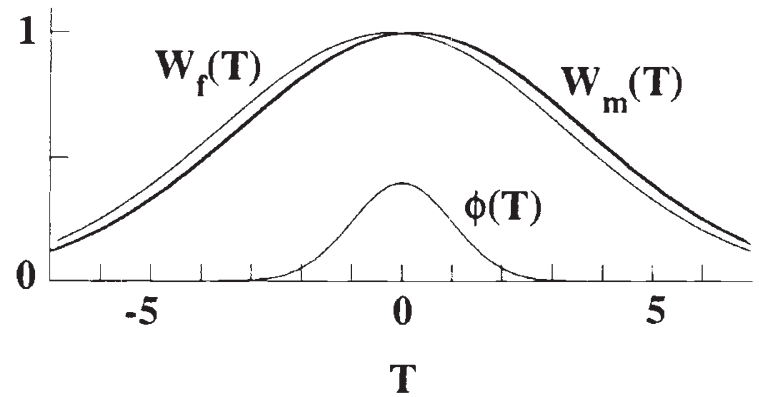

Figure 2 Fitness Functions. The $W_{m}(T)$ and $W_{f}(T)$ functions used for the results presented in fig. 1 (the male function is indicated by the heavier curve). The curve labelled $\phi(T)$ indicates the distribution of $T$ in an average year $(\bar{T}=0)$; in different years, the mean of this distribution was chosen from integer in the range -5 to 5 . The units along the horizontal axis are the same as in the left-hand diagrams of fig. 1.

chosen at random each year. The Autocorrelated- $\bar{T}$ model assumed an adult survival of 0.95 , but $\bar{T}$ was held constant for ten consecutive years before being chosen again at random.

As an illustration of the magnitude of sex differences in fitness sufficient to select extreme ESD, we observed that all variance in $Z$ was selected against in the Basic model (fig. 1, top row, the variance of $Z$ shown in this figure was maintained by mutation, as evidenced by its decrease toward zero when mutation was eliminated). The relationship between sex ratio and $T$ in this model is like that observed in many reptiles with temperaturedependent sex determination. Although not shown, increasing the difference between male and female optima facilitated the maintenance of extreme ESD, whereas reducing the difference led to a less extreme form of ESD.

Comparison of the Basic and Short-Lifespan models revealed that longevity had a major impact on the evolution of ESD (fig. 1, top tow versus middle row). Whereas all variance in $Z$ was selected against with an adult survival of 0.95 per year, a variance of $30-40$ was maintained when survival was $0 \cdot 6$ (the Short-Lifespan model). ESD is still evident at this equilibrium, but it is weak: the sex ratio changes only from $0 \cdot 3$ to $0 \cdot 8$, approximately, over four units of $T$. The effect of longevity on the evolution of ESD may be understood by realizing that fluctuations in sex ratio cancel between years in the long-lived species, hence longevity reduces the "effective" sex ratio variation induced by fluctuations in $\bar{T}$.

This interpretation of the difference between the Basic model and the Short-Lifespan model suggests the following extension. In long-lived species, if each $\bar{T}$ is allowed to persist, say, half 
of a generation before changing, the variance in $Z$ might be selected as if adult longevity was two years. This intuition was upheld qualitatively but not quantitatively: simulations of the Autocorrelated- $\bar{T}$ model showed that the variance of $Z$ evolved to a value of approximately ten when $\bar{T}$ changed every half generation, assuming an average reproductive lifespan of 20 years (fig. 1, bottom row). This variance of $Z$ substantially exceeds the equilibrium variance in the absence of autocorrelations, as in the Basic model, but it falls short of the variance selected in the Short-Lifespan model, in which $\bar{T}$ changed slightly more often than every half generation. Visually, however, this equilibrium more closely resembles the equilibrium in the Short-Lifespan model than in the Basic model. One reason why reducing generation length is not equivalent to increasing the period of environmental constancy is that the population tracks the environment during long periods of constancy.

\section{Sex ratio}

In the Basic model, extreme ESD was maintained even though more than half the years produced fewer than 20 per cent sons or more than 80 per cent sons (fig. 1, top right). Yearly primary sex ratios were less variable in the model of Autocorrelated $\bar{T}$ and were least variable in the Short-Lifespan model. The cumulative population sex ratio, which was calculated by assuming that all sex differences in fitness were incorporated into embryonic survival, remained within similar limits for all three models (fig. 1, middle column). However, the temporal pattern of variation in the cumulative sex ratio was substantially different for the three models. In the Autocorrelated $\bar{T}$ model, an excess of one sex often persisted for hundreds of generations, whereas sex ratio biases changed rapidly in the Short-Lifespan model. This property of the temporal distribution is reflected in the autocorrelations of cumulative sex ratio given in the fig. 1 (as $\rho$ ).

\section{DISCUSSION}

The present study confirms some prior conjectures about the evolution of ESD. It has been known for over ten years that environmentally-induced sex differences in fitness select ESD, provided that gender is determined in response to the sex differences in fitness (Charnov and Bull, 1977). It is also known that between-year fluctuations in the environmental determinant of sex select against ESD (Bull, 1981; Bulmer and Bull, 1982). This paper studied the evolution of ESD when both effects are present. As anticipated, the selected level of ESD decreased with the magnitude of environmental fluctuations but increased with the magnitude of sex-specific fitness effects. It was further observed that, in long-lived species, even slight sex-specific fitness effects of $T$ were sufficient to select extreme forms of ESD despite major between-year fluctuations in sex ratio, because the fluctuations tended to cancel over the length of a generation.

These results suggest that tests of adpative theories of ESD are most likely to reach definitive conclusions if conducted on short-lived species with ESD: in order to counter the disadvantages wreaked by environmental fluctuations, any advantage sufficient to maintain ESD must be large in short-lived speices. Although large advantages of ESD are not precluded in long-lived species, the failure to detect advantages of ESD may result from the existence of only weak advantages rather than from their absence. At present, proposed advantages of ESD are, to varying extents, demonstrated or plausible for amphipods, mermithid nematodes, silverside fish, and a marine worm (Naylor et al., 1988; Bull, 1983; Conover and Heins, 1987; Conover, 1984). However, there is yet no evidence to suggest a plausible advantag: of ESD in reptiles, which are of course quite longlived (Bull and Charnov, 1989).

The present results offer little solution to the female excesses observed in reptiles with ESD (Bull and Charnov, 1989). Persistence of strong excesses in the primary sex ratio is expected only in the Autocorrelated $-\bar{T}$ model, but the excess equally often favors males as females; the observed excesses instead favor only females. Strong female excesses can arise in the context of these models either if the shape of the $W_{m}(T)$ curve differs sufficiently from that of the $W_{f}(T)$ curve, or if the two curves are of similar shape but are highly asymmetric about the optimum (Bull and Charnov, 1989), so it should be realized that the absence of a highly skewed primary sex ratio in the present study resulted at least partly from our choice of male and female survival functions.

\section{REFERENCES}

AI)AMS. J., GREENWOOD, P. AND NAYL.OR, C. 1987. Evolutionary aspects of Environmental sex determination. International Journal of Invertebrate Reproduction and Development, 11, 123-136. 
BULL, J. J. 1981. Sex ratio evolution when fitness varies. Heredity, $46,9-26$.

BUll, J. J. 1983. Evolution of Sex Determining Mechanisms. Benjamin/Cummings Publ. Co., Menlo Park, CA.

BULL, J. J. 1985. Nest temperature and sex ratio in turtles: comparing field and laboratory data. Ecology, 66, 11151122 .

BULL, J. J. 1987. Evolution of phenotypic variance. Evolution, 41, 303-315.

BULL, J. J. AND CHARNOV, E. 1989. Enigmatic reptilian sex ratios. (Submitted).

BULL, J. J., VOGT, R. C. AND MCCOY, C. J. 1982. Sex determining temperatures in turtles: a geographic comparison. Evolution, 36, 326-332.

BUlmer, M. G. 1987. Sex determination in fish. Nature, 326, 440-441.

BULMER, M. G. AND BULL, J. J. 1982. Models of polygenic sex determination and sex ratio evolution. Evolution, 36, 13-26.

CHARnOV, E. L. 1982. The Theory of Sex Allocation. Princeton University Press, Princeton, N.J., USA.

CHARNOV, E. L. AND BULL, J. J. 1977. When is sex environmentally determined? Nature, 266, 828-830.

CONOVER, D. O. 1984. Adaptive significance of temperaturedependent sex determination in a fish. The American Naturalist, 123, 297-313.

CONOVER, D. O. AND HEINS, S. W. 1987. Adaptive variation in environmental and genetic sex determination in a fish. Nature, 326, 496-498.

FERGUSON, M. W. J. AND JOANEN, T. 1983. Temperaturedependent sex determination in Alligator mississippiensis. Journal of Zoology (London), 200, 143-177.

GUTZKE, W. H. N. AND PACKARD, G. C. 1985. Hatching success in relation to egg size in painted turtles (Chrysemys picta). Canadian Journal of Zoology, 63, 67-70.

HEAD, G., MAY, R. M. AND PENDLETON, L. 1987. Environmental determination of sex in the reptiles. Nature, 329, 198-199.

JOANEN, T., MCNEASE, L. AND FERGUSON, M. W. J. 1987. The effects of egg incubation temperature on post-hatching growth of American alligators. In Webb, G., Manolis, S. and Whitehead, P. (eds.) Wildlife Management of Crocodiles and Alligators. Surrey Beatty and Sons Pty. Ltd., Chipping Norton, Australia.

NAYLOR, C., ADAMS, J. AND GREENWOOD, P. J. 1988. Variation in sex determination in natural populations of a shrimp. J. Evol. Biol, 1, 355-368.

PIEAU, C. 1978. Effets de temperatures d'incubation basses et elevees, sur la differenciation sexuelle chez des embryons d'Emys orbicularis L. (Chelonien). Computes Rend. Acad. Sc. Paris (D), 286, 121-124.

TRIVERS, R. L. AND WILlARD, D. E. 1973. Natural selection of parental ability to vary the sex ratio of offspring. Science, $179,90-92$.

WEBB, G. J. W. AND SMITH, A. M. A. 1984. Sex ratio and survivorship in the Australian freshwater crocodile Crocodylus johnstoni. Symposia Zoological Society of London, No., 52, 319-355.

YNTEMA, C. L. 1979. Temperature levels and periods of sex determination during incubation of eggs of Chelydra serpentina. Journal of Morphology, 159, 17-28.

YNTEMA, C. L. AND MROSOVSKY, N. 1980. Sexual differentiation in hatchling loggerheads (Caretta caretta) incubated at different controlled temperatures. Herpetologica, 36, 3336.

\section{APPENDIX 1}

The text introduced in broad outline a model that will be described here. A summary of symbols is:

$Z \quad$ the embryonic trait influencing sex determination

$T$ the environmental variable of sex determi-

$\bar{T}_{\tau} \quad$ the average value of $T$ in year $\tau$

$\alpha(Z)$ density of $Z$ in the population

$\phi(T)$ density of $T$ in the population

$W_{m}(T)$ survival to age 1 of a male developing at $T$

$W_{f}(T)$ survival to age 1 of a female developing at $T$

\section{Inheritance of $Z$}

The character $Z$ was assumed to be affected by five, unlinked, bi-allelic loci. One locus specified the "environmental" (within-genotype) variance of $Z$, whereas the other four affected its mean. For the purpose of explanation, distinguish the two alleles at each locus as " $a$ " and " $b$ ", and designate an individual's diploid genotype $(G)$ by the fivetuplet $[l 1, l 2, l 3, l 4,15]$, where each index takes on a value of 0,1 , or 2 , and represents the number of "a" alleles at the respective locus. The "a" allele at each of loci 1-4 was assigned the phenotypic value $\mu$, the " $b$ " allele was assigned a value 0 ; at locus 5, the "a" allele was assigned a value of $\sigma_{a}^{2}$ and the "b" allele a value of $\sigma_{b}^{2}$. Each genotype, $\mathrm{G}$, was assigned a probability density of $Z$, denoted by $\alpha_{G}(Z)$, assumed to be normal with mean $M=\mu(l 1+l 2+l 3+l 4)$ and variance $V=$ $\sigma_{a}^{2}(l 5)+\sigma_{b}^{2}(2-l 5)$; this variance was necessarily non-genetic. The population variance of $Z$ thus consisted of two components, a betweenindividual component (the variance of $M$ values) and a within-individual component (the average value of $V$ ). Except for one model (designated in the text as the "Autocorrelated- $\bar{T}$ " model), the equilibrium level of variance in $Z$ was insensitive to its composition, whether within- or betweenindividual; allowing for the within-individual component enabled rapid evolution of the variance, however (cf. Bull, 1987).

\section{Variance and fitness effects of $T$}

Whereas the mean and variance of $Z$ were allowed to evolve, the character $T$ was not allowed to evolve. The density of $T$ was assumed to be normal 
with mean $\bar{T}_{\tau}$ and variance of unity in generation $\tau$. The mean was itself chosen as a random variable to mimic environmental fluctuations (see below for details). The choice of a variance of unity was based on the single study available that provides information on this quantity for a natural population (Bull, 1985), which was based on 75 nests of map turtles on a single nesting beach during one summer. Using either the mean nest temperature or Bull's "corrected" temperature as the measure of $T$, the standard deviation of temperature observed in the original data is between 1 and $2{ }^{\circ} \mathrm{C}$. This value applies to the between-nest variance of temperature, so the between-egg variance in temperature would be even larger. (In our model, the variance in $T$ is most appropriately regarded as the between-egg variance in temperature.) In view of the fact that large variances in $T$ minimize the effect of between-year fluctuations in $\bar{T}$ and thus facilitate the maintenance of ESD (unpublished results), we chose a variance of unity as a conservatively low value.

The male and female survival functions, $W_{m}(T)$ and $W_{f}(T)$, were treated as densityindependent viabilities. Both functions were assumed to follow Gaussian or normal form. For Gaussian functions, survival declines as $T$ deviates from the optimum $(\gamma)$ according to $\left\{\exp -\frac{1}{2}\left[(T-\gamma)^{2} / \omega^{2}\right]\right\}$, assuming that survival at the optimum is unity. The span $\omega$ determines the curvature of this function: small values of $\omega$ indicate that viability falls off rapidly as $T$ deviates from the optimum. Survival is never allowed to reach zero, but it falls from $W=1$ at the optimum to $W=0.6$ at $1 \omega$ from the optimum, to $W=0.13$ at $2 \omega$ from the optimum (e.g., fig. 2).

In reptiles, the reported viability effects of incubation temperature certainly depart from Gaussian in that viability is zero at extremes, and viability effects may also be asymmetric, but the Gaussian assumption is probably adequate for our purposes. Work on a variety of turtles with ESD has shown that development is viable over a range of perhaps $10^{\circ} \mathrm{C}$ (e.g., Pieau, 1978; Yntema, 1979; Yntema and Mrosovsky, 1980; Bull et al., 1982; Gutzke and Packard, 1985), whereas crocodilians tolerate a narrower range (Ferguson and Joanen, 1983; Webb and Smith, 1984; Joanen et al., 1987). We thus chose the value $3 \cdot 5$ for the spans of male and female survival functions. As there is no evidence that the effects of extreme temperatures on viability differ for males and females, we modeled sex differences in fitness merely as differences in the optima between $W_{m}(T)$ and $W_{f}(T)$. The optima were set at $+0 \cdot 2$ and $-0 \cdot 2$ values of $T$.

\section{Life cycle}

The life cycle consisted of the following order of events. With $M$ adult males and $F$ adult females in year $\tau$, gametes were formed, each male producing an infinite number of sperm and each female producing a fixed number of eggs. Female fecundity was calculated deterministically and was chosen so that the population size did not change much in the long term. Mutation occurred at loci 1-4 in gametes, each allele mutating to the alternative state with probability $10^{-5}$ and remaining unchanged otherwise (mutation was not allowed at locus 5 , the locus affecting within-genotype variance in $Z$ ). After mutation, male gametes united (randomly) with female gametes to form zygotes. For each zygote, genotype was specified for all loci, and gender was determined according to its distribution of $Z$ (which in turn depended on genotype) and by the environmental distribution of $T$. Survival of each genotype was specified according to gender and to the distribution of $T$. The number of adult females in year $\tau+1$ was then calculated as the sum of two terms: (i) the adult females surviving from year $\tau$, and (ii) the surviving female newborns; adult survival was independent of $T$ and sex. The recursion for males was similar.

The environmental state was varied between years by varying $\bar{T}$, which was chosen at random from eleven possible discrete states (spaced evenly along $T$ ); $\bar{T}$ was chosen as a continuous variable from a normal distribution, with mean zero and variance unity, and was then converted to an integer in the range $[-5,5]$ by truncating or eliminating the remainder. These values were chosen because they yielded acceptably large fluctuations in the primary sex ratio of the Basic model (fig. 1); empirical guidance on these parameters is lacking. The long term mean of $\vec{T},(0)$, was set equidistant between the male and female optima.

For a zygote (genotype $G$ ) conceived in year $\tau$, its expected contribution to the adult male pool in year $\tau+1$ was calculated as

$$
\int_{-\infty}^{\infty} \phi(T) W_{m}(T) \int_{-\infty}^{T} \alpha_{G}(Z) \mathrm{d} Z \mathrm{~d} T,
$$

and its contribution to the adult female pool was calculated as

$$
\int_{-\infty}^{\infty} \phi(T) W_{f}(T) \int_{T}^{\infty} \alpha_{G}(Z) \mathrm{d} Z \mathrm{~d} T,
$$

where the mean of $T$ in the distribution $\phi(T)$ was taken from year $\tau$. Zygotes were then produced by each adult genotype according to its proportional representation in the male-gamete and femalegamete pools. 